\begin{tabular}{|c|c|c|c|c|c|}
\hline MUNIBE Antropologia-Arkeologia & $n^{\circ} 71$ & $193-205$ & DONOSTIA & 2020 & ISSN 1132-2217 • eISSN 2172-4555 \\
\hline
\end{tabular}

\title{
Prácticas ganaderas en el asentamiento rural de La Cruceta (Tauste, Zaragoza) durante los siglos XIII y XIV d.C.
}

\author{
Animal husbandry practices in the rural settlement of La Cruceta \\ (Tauste, Zaragoza) during the 13th and 14th centuries AD.
}

PALABRAS CLAVES: Arqueozoología, Baja Edad Media, Ganadería, Reino de Aragón.

GAKO-HITZAK: Arkeozoologia, Behe Erdi Aroa, Abeltzaintza, Aragoiko Erreinua.

KEY WORDS: Archaeozoology, Late Middle Ages, Animal husbandry, Kingdom of Aragon.

\section{Alejandro SIERRA SAINZ-AJA(1), Fco. Javier GUTIÉRREZ GONZÁLEZ(2), Víctor GIL DE MURO EGUIZÁBAL(2), Óscar LANZAS ORENSANZ( ${ }^{(2)}$, Carlos VALLADARES LAFUENTE ${ }^{(2)}$, Mario LAFUENTE GÓMEZ ${ }^{(3)}$}

\section{RESUMEN}

La Cruceta es un yacimiento situado en Tauste, en la provincia de Zaragoza. Se trata de un asentamiento rural, que cumplía las funciones de puesto de vigilancia, ocupado entre los siglos XIII y XIV d.C. Durante su excavación, se documentaron estructuras de habitación y de funciones auxiliares, así como cerámica decorada, monedas y elementos metálicos de indumentaria militar. Además, se recuperó una abundante cantidad de restos faunísticos. En este trabajo, se presenta el estudio arqueozoológico de La Cruceta, uno de los primeros para reino de Aragón en época medieval. Los resultados han permitido documentar, además de una funcionalidad estratégica, la existencia de una economía centrada en el ganado ovino y caprino. Los productos explotados son variados (carne, leche, materias primas, etc.), lo que junto a las pautas de procesado parece indicar que La Cruceta, además de puesto de vigilancia, tuvo funciones de producción.

\section{LABURPENA}

La Cruceta Tauste udalerrian (Zaragoza probintzia) kokatutako aztarnategi bat da. Zaintza-postuaren funtzioak betetzen zituen landa-eremuko giza kokaleku bat da, K.o. XIII. eta XVI. mendeen artean okupatutakoa. Indusketa egin zenean, bizitzeko eta funtzio osagarrietarako egiturak dokumentatu ziren, hala nola zeramika dekoratua, txanponak eta jantzi militarretako elementu metalikoak. Gainera, faunaren aztarna ugari ere berreskuratu ziren. Lan honetan La Crucetaren (Aragoiko Erreinuko Erdi Aroko lehen aztarnategietako bat) ikerketa arkeozoologikoa aurkeztuko da. Emaitzek aztarnategian garai hartan ardi- eta ahuntz-aziendaren bueltako ekonomia zutela dokumentatu dute. Hainbat produktu ustiatzen zituzten (haragia, esnea, lehengaiak, etab.), eta badirudi horrek eta prozesatzeko erabiltzen zituzten moduek adierazten dutela La Crucetak, zaintza-postuaren funtzioa ez ezik, ekoizpen-funtzioak ere bazituela.

\section{ABSTRACT}

The Kingdom of Aragon expanded territorially during the 12th and 13th centuries. This expansion was linked to the growth and development of the Aragonese economy, being the agricultural activities the predominant ones. Aragonese husbandry was linked to this territorial and economic expansion of the Kingdom of Aragon, with the increase of the livestock population that took place throughout the 13th century. Studies on this period in the Kingdom of Aragon are practically non-existent, due to the absence of local specialists for this type of study and the scarce interest that has traditionally been given to it.

Within this context we find the site of La Cruceta, a Late Medieval settlement located in the municipality of Tauste, in the province of Zaragoza. This settlement was occupied between the beginning of the 13th century and the middle of the 14th century, and it is not clear who promoted this construction. Documentary sources have led to the conclusion that it could have been promoted by the Santa Cristina de Somport Hospital, by the Casa de los Luna or by the municipality of Tauste within a climate of rivalry for the control and exploitation of natural resources. The aims of this work are: 1) to document livestock practices and 2) to show the livestock products exploited by those who occupied La Cruceta, seeking to characterise the economic strategy carried out there.

The results have shown how the inhabitants of La Cruceta had a livestock focused on the exploitation of sheep and goats. The dominance of these two species can be observed during most of the Middle Ages in the Iberian Peninsula, but especially from the 13th century onwards, when they formed $50 \%$ of the domestic livestock. Furthermore, pigs, cattle, horses and birds also made up the livestock in La Cruceta, although with much lesser quantitative importance. Finally, hunting of species such as red deer also formed part of the economic activities of the site. The results have also shown how the community that occupied La Cruceta exploited a great diversity of animal products (meat, milk, raw materials), highlighting the limited role that wool exploitation could have played. In addition, the presence of perinatal animals led to the

\footnotetext{
(1) Grupo Primeros Pobladores del Valle del Ebro, Universidad de Zaragoza - Laboratori d'Arqueozoologia, Universitat Autònoma de Barcelona. asierrasainzaja@hotmail.com. Avda. Cataluña $1813^{\circ}$ I 50014 Zaragoza.

(2) Arqueólogo profesional.

(3) Departamento de Historia, Universidad de Zaragoza.
} 
conclusion that they were bred at the site. With regard to butchering, the absence of elements such as humerus and femurs from sheep and goats, with a high meat content, stands out.

In conclusion, our hypothesis is that in addition to being a strategic settlement for the control of the area, La Cruceta was also a settlement with livestock production for the self-consumption of the community that inhabited the site and perhaps for the supply (mainly of meat) of some settlement near the site. This hypothesis is based on the specialization of production in sheep and goats, the mortality profile of both species and the representation of the anatomical regions, which shows a notable absence of elements with higher meat content in these species.

\section{INTRODUCCIÓN}

El reino de Aragón se expandió territorialmente durante los siglos XII y XIII. Esta expansión vino de la mano del crecimiento y desarrollo de la economía aragonesa, siendo las actividades agropecuarias las predominantes (Ledesma, 1989). A su vez, la ganadería aragonesa estuvo ligada a esta expansión territorial y económica del reino de Aragón, con el aumento de la cabaña ganadera que se produjo a lo largo del siglo XIII (Sarasa 1989). Este crecimiento de la ganadería está vinculado a la consolidación de instituciones como las casas de ganaderos, los ligallos y las cofradías de pastores (Ledesma, 1989, Fernández Otal, 1996). La producción ganadera se orientó tanto al autoconsumo como a la comercialización. Productos como la carne, la leche o la lana eran consumidos localmente pero también se exportaban a las ciudades y fuera del reino. La lana es un buen ejemplo, ya que su industria tuvo un importante papel dentro de la economía aragonesa en estos siglos (Sesma, 1977, 1995 y 2009; Navarro, 2003). La carne, otro. Este producto se convertiría en una parte fundamental de la dieta urbana, como lo atestigua el surgimiento de las agrupaciones de carniceros (Sarasa, 1995; Falcón, 1998).

Estos aspectos de la economía aragonesa durante la Edad Media han sido estudiados exclusivamente a partir de las fuentes escritas, pero la arqueología puede tener un papel importante en el estudio de los mismos. Concretamente, la arqueozoología puede aportar información sobre las prácticas ganaderas y alimenticias en el Reino de Aragón durante la Edad Media. La arqueozoología medieval ha tenido un corto desarrollo en la Península Ibérica (Grau y Garcia, 2018). Pocos son los trabajos existentes para este periodo, aunque en los últimos años han ido en aumento en algunas regiones como País Vasco, Madrid, Cataluña o Andalucía oriental (Vigil-Escalera et al. 2014; Valenzuela-Lamas et al. 2014; Grau-Sologestoa et al. 2016; Grau-Sologestoa 2017; García-García 2017). Por el contrario, en regiones como Aragón, la ausencia de estudios arqueozoológicos es casi total. Tan solo contamos con los estudios del Castillo de Albarracín (Moreno García, 1999), de las Sillas de Marcén (Sénac, 2009) y de Zafranales (Castaños, 1988). Esta escasez de estudios se ha debido, fundamentalmente, a la ausencia de especialistas locales para este tipo de estudios y al escaso interés que se le ha dado tradicionalmente a los mismos.

Dentro de este contexto encontramos el yacimiento de La Cruceta (Fig. 1), un asentamiento bajomedieval de vigilancia situado en el término municipal de Taus- te, en la provincia de Zaragoza. Este asentamiento fue ocupado entre comienzos del siglo XIII y mediados del XIV, no estando claro quién impulsó esta construcción. Las fuentes documentales han llevado a plantear que pudo ser impulsada por el Hospital de Santa Cristina de Somport, por la casa de los Luna o por el municipio de Tauste dentro de "un clima de rivalidad por el control y explotación de los recursos naturales, especialmente los pastos y el monte, dando lugar a tensiones e incluso enfrentamientos armados, en los que pudieron participar tanto los agentes del poder señorial como las propias comunidades campesinas" (Gutiérrez González et al., 2019: 55), en el que La Cruceta pudo suponer un enclave estratégico en altura y un lugar desde el que guardar el ganado. Con este bagaje, planteamos como objetivos de este trabajo: 1) documentar las prácticas ganaderas y 2) evidenciar los productos ganaderos explotados por quienes ocuparon La Cruceta, buscando caracterizar la estrategia económica llevada a cabo en el mismo.

\section{MATERIALES Y MÉTODOS}

Tras las tres campañas efectuadas, se han excavado al menos 12 espacios distintos (Fig. 2). En la parte alta del cerro se han comprobado una serie de estancias que suponemos de reposo, a las que se accedía a través de un espacio distribuidor (Espacio 3), al que se accedería a su vez desde el Este, en una primera fase, a través de la puerta existente entre los Muros 3 y 5, que en un momento posterior fue tapiada (Gutiérrez González et al., 2017, 2019).

A lo largo de la ladera se abren distintos espacios adosados a los laterales de los muros perimetrales, con una disposición transversal. Entre los Espacios 6 y 7 se han identificado lo que parecen las esquinas de acceso en una primera fase de ocupación, cuyo espacio debió ser ampliado y reformado probablemente a fines del siglo XIII o principios del siglo XIV. En el resto de espacios documentados hasta la fecha en la ladera no se han apreciado características definitorias de su función.

El conjunto de fauna está formado por un total de 497 restos, procedentes prácticamente en su totalidad de los niveles de abandono de la ocupación de la zona baja de la ladera (espacios 6 a 10), pues en la zona alta apareció muy poco material sobre los suelos o en los derrumbes de relleno. La recogida del material se llevó a cabo a mano, con el cribado de algunas áreas concretas. A pesar de ello, el número de elementos de pequeño tamaño es abundante, incluyendo restos de animales perinatales. 


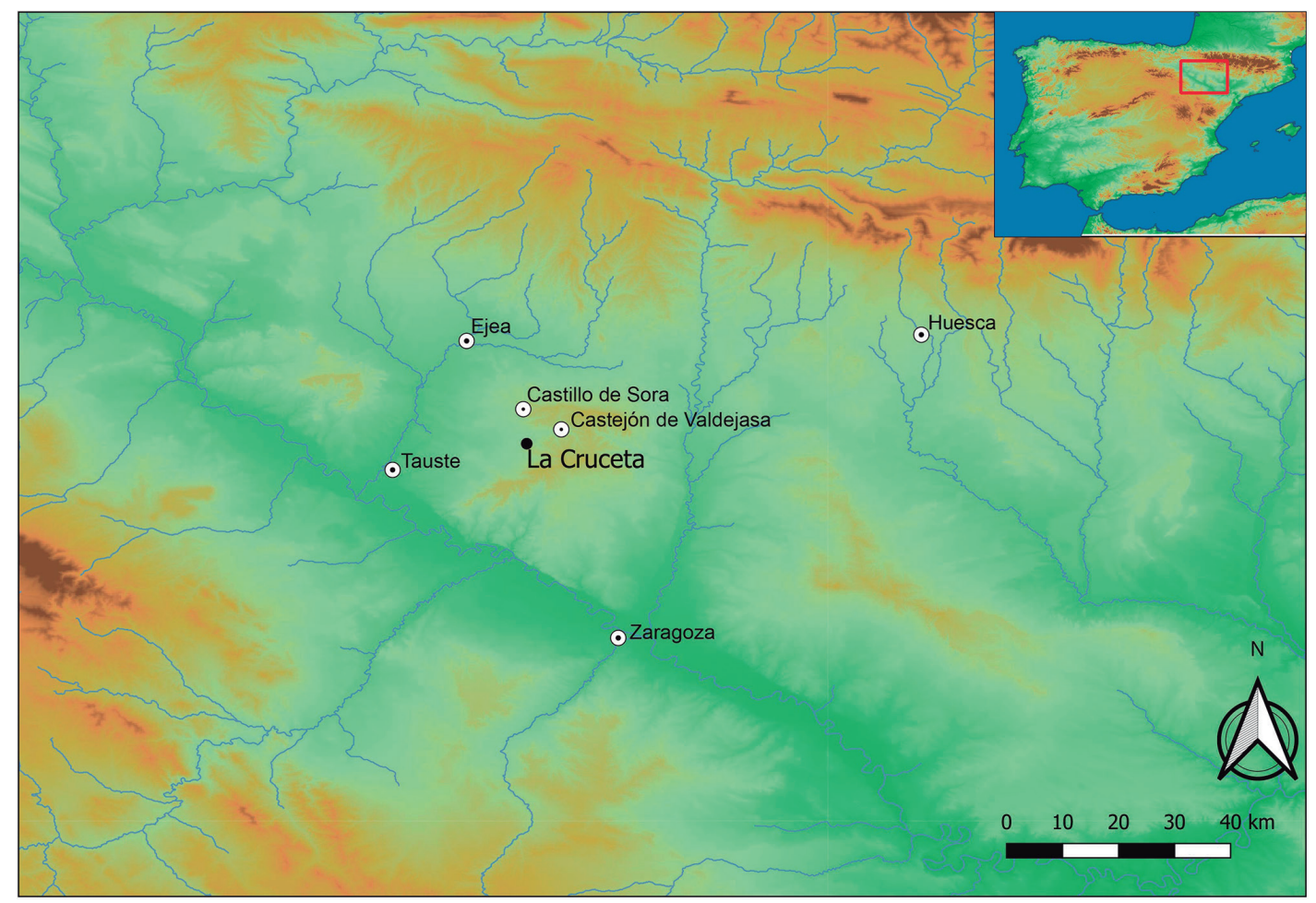

Fig.1. Mapa de situación del yacimiento (autor: Alejandro Sierra). / Location map of the site (author: Alejandro Sierra).

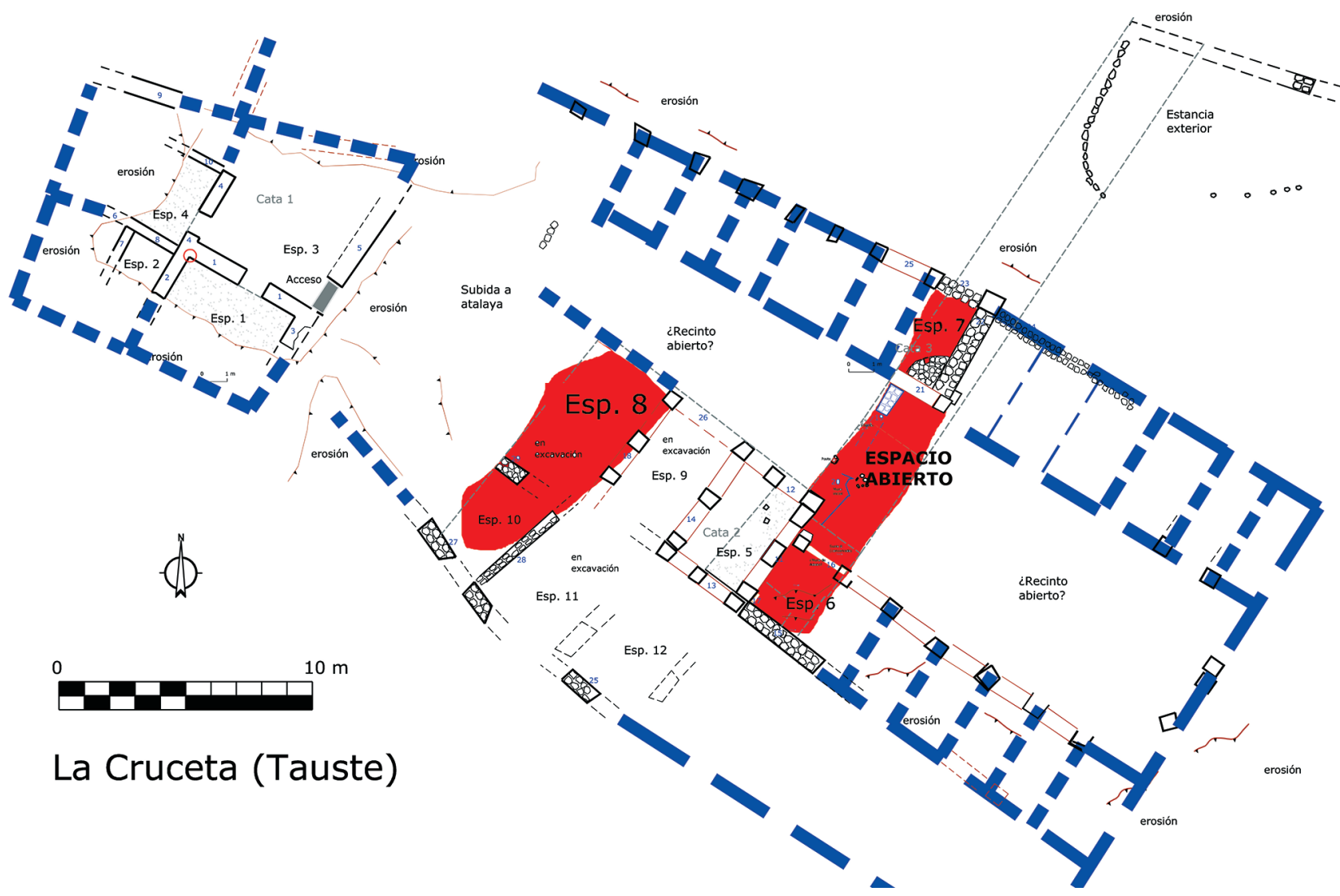

Fig.2. Plano del sitio con las catas y los espacios excavados con las zonas de procedencia de la mayor parte de la fauna marcadas en rojo (autor: Fco. Javier Gutiérrez González). / Map of the site with the excavated areas and with the areas of origin of most of the fauna marked in red (author: Fco. Javier Gutiérrez González). 
Metodológicamente, se realizó un análisis arqueozoológico con un análisis anatómico y taxonómico utilizando las colecciones de referencia del Laboratorio de Prehistoria y Arqueología de la Universidad de Zaragoza. Además, se han utilizado diferentes atlas de anatomía comparada: Schmidt (1972), Barone (1969), Pales y Lambert (1970), Lavocat (1967), Fernández (2001), Varela y Rodríguez (2004) y Cohen y Serjeantson (1996). Se han utilizado diferentes publicaciones con el objetivo de distinguir a la oveja y la cabra. Estas publicaciones se dividen en dos tipos, las que presentan criterios para dientes (Payne, 1985; Helmer, 2000; Halstead el al., 2002; Balasse y Ambrose, 2005; Zeder y Pilaar, 2010; Gillis et al., 2011) y las que los presentan para los elementos del esqueleto postcraneal (Boessneck et al., 1964; Boessneck, 1970; Prummel y Frisch, 1986; Zeder y Laphalm, 2010).

La edad de los caprinos domésticos se ha calculado con los restos dentarios a partir de las propuestas de Payne (1973 y 1987), complementadas por Helmer (1995). La edad a partir de la fusión epifisaria se ha calculado utilizando las indicaciones de Zeder (2006). Para la elaboración de los histogramas se aplicó la metodología de Gerbault et al. (2016). En el caso del ganado bovino, se ha utilizado la propuesta de Grant (1982), utilizando las clases de edad establecidas por Legge (1992), para los restos dentarios. El estudio de la fusión epifisaria se ha basado en los datos aportados por Silver (1969). Para el ganado porcino se han empleado los trabajos publicados recientemente por Zeder y colegas (Lemoine et al., 2014; Zeder et al., 2015). Para calcular la edad de las especies salvajes, como el ciervo, se han utilizado diferentes métodos (Mariezkurrena, 1983; Azorit et al., 2002, Brown y Chapman, 1991).

El modelo de interpretación de las trazas y los patrones de fracturación es el planteado por Saña (1999), derivado de los trabajos de Binford (1981), Diez (1992) y Lyman (1994), en el que se distinguen: despellejado (extracción de la piel), evisceración (extracción de las vísceras), descuartizado (división del animal en cuartos), desmembrado (separación de las articulaciones), descarnado (extracción de la carne) y aprovechamiento medular. Para ello, se analizan las trazas de corte, su dirección, su tipo, su función, etc. Las trazas de corte se han definido según su morfología siguiendo trabajos como los de Pérez-Ripoll (1992) o Potts y Shipman (1981). La fracturación ha sido analizada teniendo en cuenta el trabajo de Vila y Mahieu (1991), que permite clasificar fracturas según su ángulo, su perfil y su borde. Se ha incluido un anexo con las medidas en bruto.

\section{RESULTADOS}

El conjunto está compuesto por 497, de los cuales 254 han podido ser identificados anatómica y taxonómicamente, lo que supone un 51,1\% del total de restos. Los restos se encuentran bien conservados, documentándose elementos infantiles entre ellos. Las marcas de raíces y las concreciones son las principales marcas naturales documentadas. Ha podido ser detectada la presencia de marcas de carnívoros, indicando la intervención de estos animales sobre una parte de los huesos. Un total del 3.9\% de los huesos aparecen con este tipo de marcas, que se localizan normalmente en los extremos de las diáfisis de los huesos largos.

El espectro faunístico muestra una clara preferencia por los animales domésticos (Tabla I). El ganado ovino y el caprino dominan con más del 80\% del NISP y del 60\% del NMI, pero tan solo una parte ha podido ser identificada como perteneciente a oveja o a cabra, lo que dificulta la interpretación de la composición de los rebaños. Los restos identificados muestran un predominio del ganado caprino sobre el ovino de una proporción de 2,5:1 (Tabla II). Por detrás, en pequeño porcentaje, aparecen otras especies de mamíferos domésticos que complementarían el rebaño, como la vaca y los équidos. En cuanto a los suidos, la talla de los mismos, junto a la presencia de animales muy jóvenes y el contexto en el que nos encontramos, han sido identificados como cerdos domésticos. Además, se documenta la actividad

\begin{tabular}{|c|c|c|c|c|}
\hline & NISP & \%NISP & NMI & \%NMI \\
\hline Bos taurus & 4 & $1.6 \%$ & 2 & $5.3 \%$ \\
\hline Ovis/Capra & 171 & $67.3 \%$ & & $0.0 \%$ \\
\hline Ovis aries & 13 & $5.1 \%$ & 6 & $15.8 \%$ \\
\hline Capra hircus & 27 & $10.6 \%$ & 15 & $39.5 \%$ \\
\hline Sus domesticus & 12 & $4.7 \%$ & 4 & $10.5 \%$ \\
\hline Equus $s p$ & 2 & $0.8 \%$ & 1 & $2.6 \%$ \\
\hline Cervus elaphus & 6 & $2.4 \%$ & 1 & $2.6 \%$ \\
\hline Oryctolagus cuniculus & 8 & $3.1 \%$ & 1 & $2.6 \%$ \\
\hline Anátida & 1 & $0.4 \%$ & 1 & $2.6 \%$ \\
\hline Columbiforme & 1 & $0.4 \%$ & 1 & $2.6 \%$ \\
\hline Corvus corax & 2 & $0.8 \%$ & 1 & $2.6 \%$ \\
\hline Gallus gallus & 1 & $0.4 \%$ & 1 & $2.6 \%$ \\
\hline Galliforme & 3 & $1.2 \%$ & 2 & $5.3 \%$ \\
\hline Alectoris rufa & 2 & $0.8 \%$ & 1 & $2.6 \%$ \\
\hline Avifauna & 1 & $0.4 \%$ & 1 & $2.6 \%$ \\
\hline TOTAL NISP & 254 & & 38 & \\
\hline M. Grande & 12 & & & \\
\hline M. Medio-Grande & 2 & & & \\
\hline M. Medio & 208 & & & \\
\hline M. Medio-Pequeño & 1 & & & \\
\hline M. Pequeño & 1 & & & \\
\hline Indeterminado & 19 & & & \\
\hline TOTAL & 497 & & & \\
\hline
\end{tabular}

Tabla 1: Representación taxonómica de La Cruceta. / Taxonomic representation of La Cruceta.

\begin{tabular}{|c|c|c|}
\cline { 2 - 3 } \multicolumn{1}{c|}{} & NISP & NMI \\
\hline Oveja & $32.5 \%$ & $28.6 \%$ \\
\hline Cabra & $67.5 \%$ & $71.4 \%$ \\
\hline
\end{tabular}

Tabla 2: Proporción de ovejas y cabras de La Cruceta. / Proportion of sheep and goats from La Cruceta. 
cinegética con especies como el ciervo, representadas con muy pocos restos. El conejo también tiene una presencia testimonial, aunque no ha sido posible saber si era salvaje o doméstico. Lo que sí que parece que fueron los humanos los que aportaron los restos, ya que en una parte de los mismos han sido documentadas marcas antrópicas. Por último, se detecta una gran variedad de especies de aves representadas con escasos restos, como gallinas, perdices, cuervos o palomas. Algunos de estos restos han podido ser sólo identificados a nivel de orden. Se identifican restos de galliformes, que pueden pertenecer a especies como gallinas, pavos, urogallos, faisanes, etc, aunque probablemente se trate de restos de gallina, debido a la elevada frecuencia de esta especies en los yacimientos de época medieval. También se encuentra un resto de anátidas dentro del conjunto, pudiendo tratarse de Anas.

En cuanto a los restos identificados sólo anatómicamente, se observa un claro dominio de los restos de mamíferos de tamaño medio, muy posiblemente oveja o cabra, ya que son los que predominan en el conjunto. La mayoría de los restos clasificados en esta categoría pertenecen a restos de costillas o de diáfisis de hueso largo que no han podido ser determinadas taxonómicamente.

En lo relativo a la edad de sacrificio, sólo ovejas y cabras aportan una buena muestra. El resto de especies son escasas pero se observan diferencias entre ellas. En el ganado vacuno, los dos individuos identificados pertenecen a un individuo fetal y a otro sacrificado entre los 7 y los 10 meses. El ganado porcino aparece representado con dos indivuduos fetales, uno mayor de 24 meses y otro entre 30 y 72 meses. En el caso del ganado equino se documenta un individuo de más de 36 meses. El único individuo de ciervo documentado fue cazado

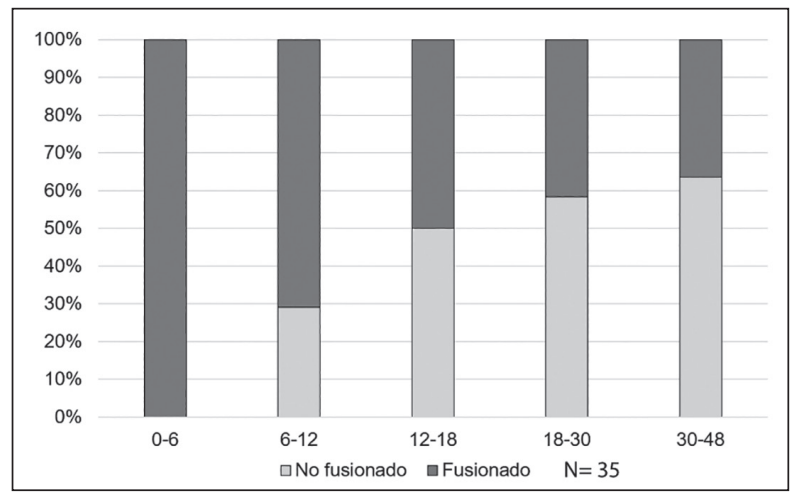

Fig.3. Edad de fusión epifisaria de las ovejas y las cabras (en meses) a partir del NISP. / Age of epiphyseal fusion of sheep and goats (in months) from NISP.

con 32 meses aproximadamente. La mayor parte de las aves son sacrificadas a edad adulta. Tan sólo uno de los individuos de galliformes pertenece a un individuo inmaduro. La diferenciación sexual ha sido imposible, únicamente en el caso de las gallinas se ha identificado un macho debido al espolón del tarso-metatarso.

En cuanto a ovejas y cabras, tanto la fusión epifisaria como la erupción y el desgaste dentario aportan información. La fusión epifisaria (Fig. 3), que ha tenido que ser calculada con ovejas y cabras juntas, muestra como una buena parte de Ovis/Capra son sacrificados antes de los 18-30 meses, predominando los sacrificios a partir de los 12 meses. Una parte de los rebaños se sacrifican a partir de los 30-48 meses. En el caso de los dientes, donde han podido separarse las ovejas y las cabras, se observan diferencias entre ambas especies (Fig. 4 y 5). Por un lado, las cabras son sacrificadas entre los 2 y los 6 meses, justo después del destete, y

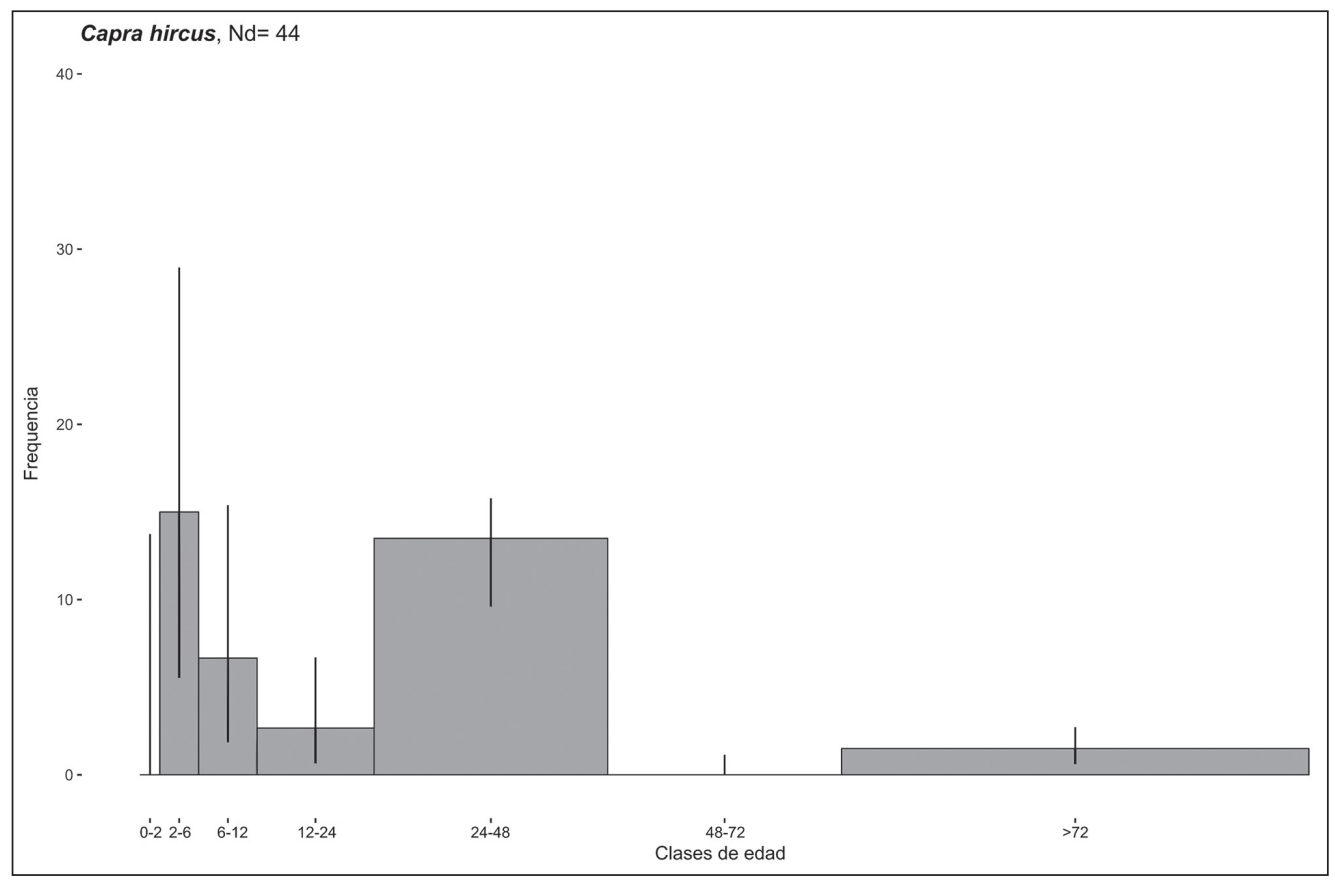

Fig.4. Histograma de edad de la cabra a partir del desgaste dental $(\mathrm{Nd}=$ número de dientes). / Histogram of goat age from tooth wear $(\mathrm{Nd}=$ number of teeth). 


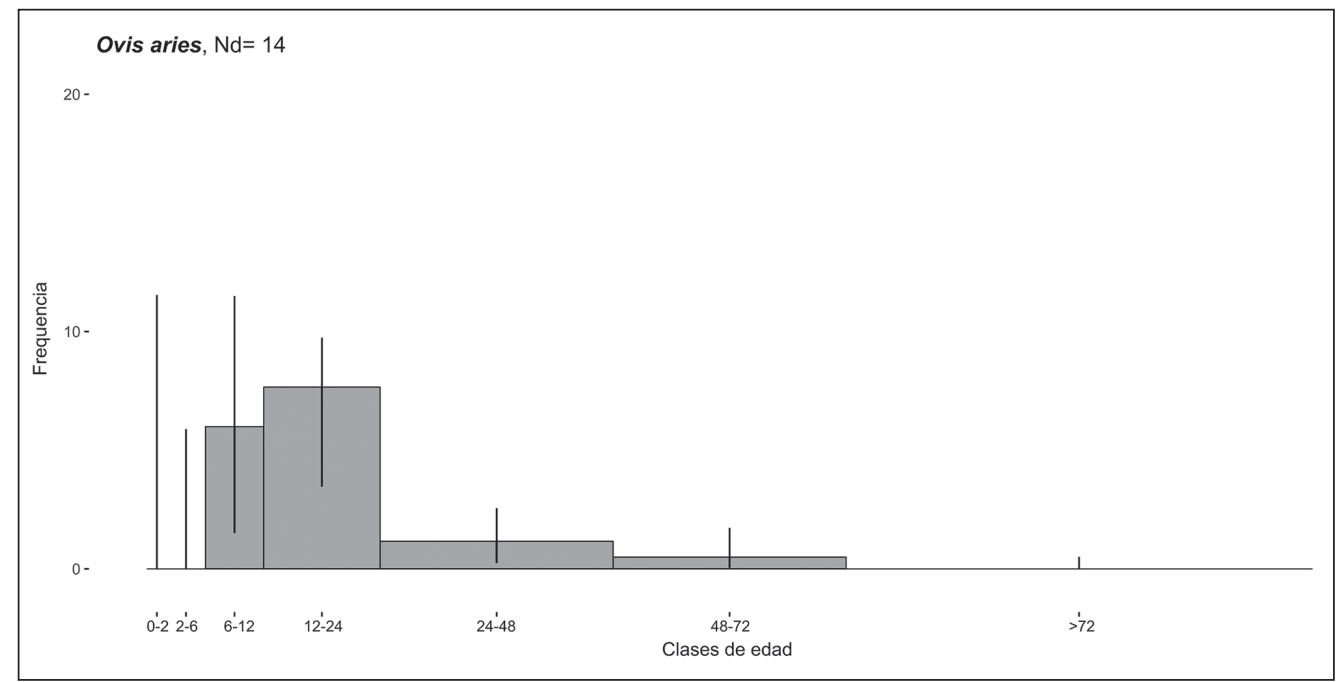

Fig.5. Histograma de edad de la oveja a partir del desgaste dental $(\mathrm{Nd}=$ número de dientes). / Histogram of sheep age from tooth wear ( $\mathrm{Nd}=$ number of teeth). entre los 24 y 48 meses. Por otro lado, aunque la muestra es menor, las ovejas son sacrificadas mayoritariamente entre los 6 y los 24 meses. La comparación de la edad dentaria y epifisaria muestra tendencias similares, como los sacrificios a partir de los 12-18 meses, pero se observan diferencias como la ausencia de restos de animales sacrificados antes de los 6 meses o el gran porcentaje de animales sacrificados a partir de los 3048 meses.

La abundancia de restos de ovejas y cabras también ha permitido identificar parcialmente la composición sexual del rebaño. A través de los restos de pelvis, han podido ser identificadas al menos tres hembras y un macho, lo que hace suponer un mayor porcentaje de hembras en el rebaño, seguramente debido a intereses productivos. La escasez de medidas ha impedido realizar una aproximación biométrica a la distribución sexual de los rebaños.

La distribución anatómica ha sido prácticamente imposible de analizar en la mayor parte de las especies debido a la escasez de restos (Tabla III). En el caso del ganado bovino y el equino, se encuentran escasos cua-

\begin{tabular}{|l|c|c|c|c|}
\cline { 2 - 5 } \multicolumn{1}{c|}{} & BOTA & SUDO & EQSP & CEEL \\
\hline Cráneo & & 2 & & 3 \\
\hline Axial & 1 & & & 2 \\
\hline Ext. Anterior superior & & 4 & & \\
\hline Ext. Anterior inferior & 2 & 3 & & \\
\hline Ext. Posterior superior & & 2 & & \\
\hline Ext. Posterior inferior & 1 & & 2 & \\
\hline Ext. Distal & & 1 & & 1 \\
\hline
\end{tabular}

Tabla 3: Distribución anatómica de Bos taurus (BOTA), Sus domesticus (SUDO), Equus caballus (EQCA) y Cervus elaphus (CEEL). / Anatomical distribution of Bos taurus (BOTA), Sus domesticus (SUDO), Equus cabaIlus (EQCA) and Cervus elaphus (CEEL). tro y dos restos respectivamente. Del ganado porcino, están prácticamente representadas todas las partes anatómicas. Respecto al ciervo, se documentan restos del cráneo, pertenecientes dos astas y un maxilar, del esqueleto axial (costilla y vertebra) y de la parte inferior de las extremidades, correspondiente a una falange. Tan sólo ha podido realizarse un análisis más amplio en el caso de ovejas y cabras (Fig. 6), aunque no se ha distinguido entre ambas especies. Resulta destacable la abundancia de ciertos huesos como los radios, las tibias y las mandíbulas, que suponen más del 30\% de todos los restos a pesar de no ser los huesos más representados en un animal completo. Otros elementos como la escápula, la pelvis o las costillas también aparecen representados con más de un 5\% del total de restos. En contraste, elementos como el húmero y el fémur apenas aparecen representados. Si comparamos el número de restos identificados con la densidad de los huesos (loannidou 2003) para caracterizar la conservación diferencial (Fig. 7), observamos cómo hay una baja correlación entre la presencia de los distintos elementos y su densidad $\left(r=0,612 ; r^{2}=0,374\right)$. Los elementos de mayor densidad como son las diáfisis mediales del radio y la tibia están sobrerrepresentados. Sin embargo, otros elementos como las partes distales de los húmeros, los metacarpos y los isquiones y los iliones de las pelvis que están infrarrepresentados respecto a su densidad. Otros elementos como las escápulas (salvo fosa articular) y las diáfisis distales de los radios también se encuentran sobrerrepresentados respecto a su baja densidad. Además, el radio y la tibia están más representados que otros elementos apendiculares como escápulas, húmeros, metacarpos, fémures y metatarsos, aunque están igual representados en el esqueleto (Fig. 8). Destaca también la escasa representación (salvo los metatarsos) de las partes distales de las extremidades (carpales, tarsales, metacarpos y falanges), que son las partes de menos contenido cárnico, aunque esto podría deberse a la recogida a mano 


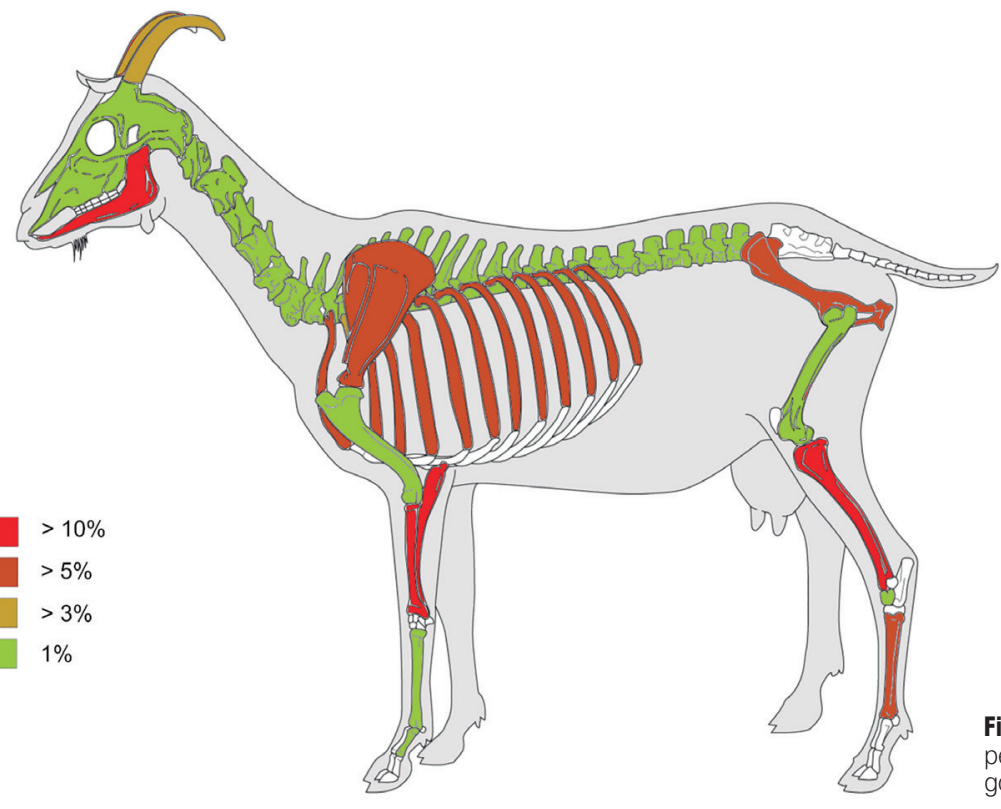

Fig.6. Distribución anatómica de ovejas y cabras respecto al NISP. / Anatomical distribution of sheep and goats with respect to NISP.
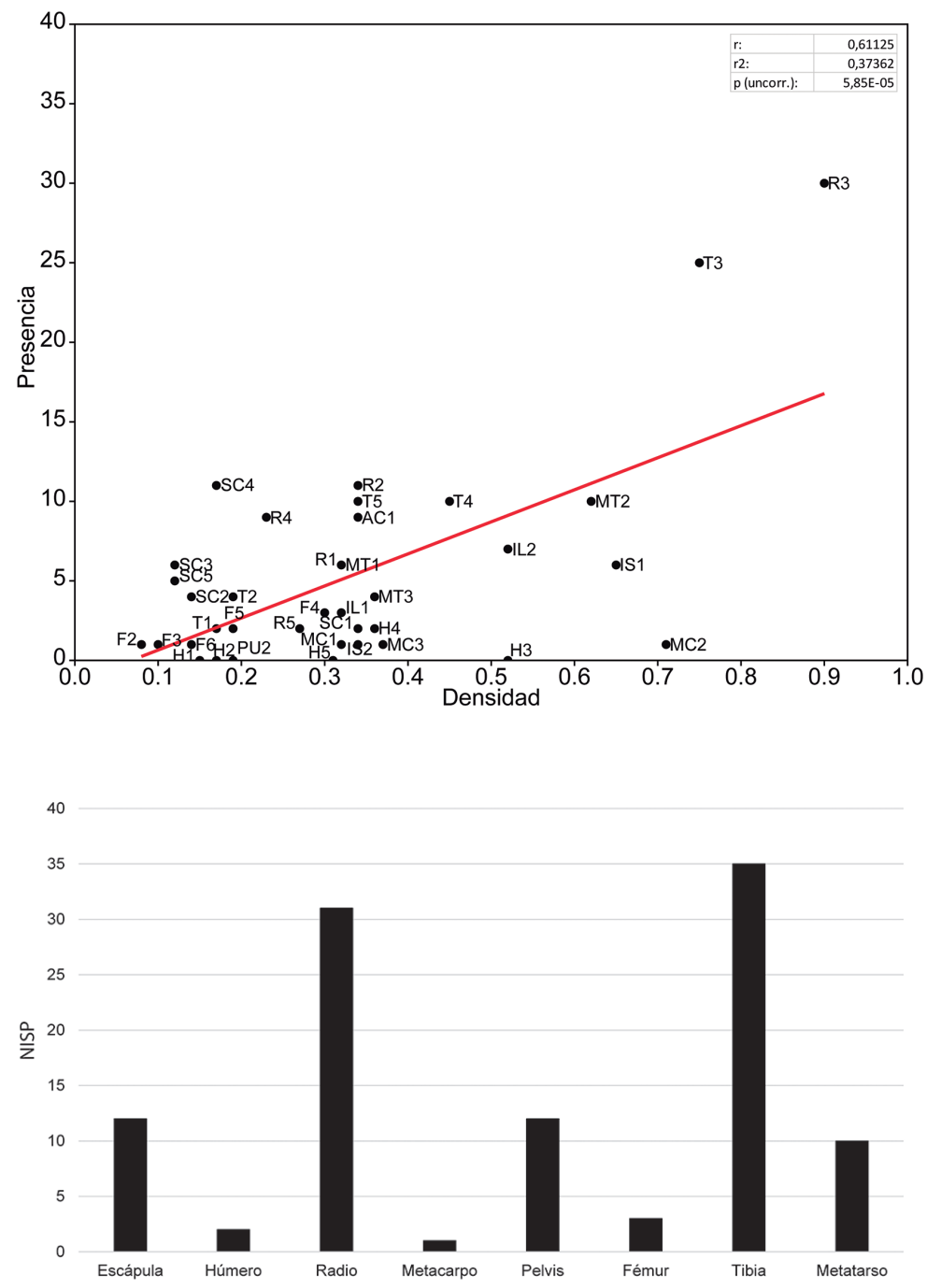

Fig.7. Correlación entre la densidad y la presencia (NISP) de los elementos de las extremidades de oveja y cabra. / Correlation between density and presence (NISP) of sheep and goat limb elements. 
del material, si bien es cierto, que otros elementos de pequeño tamaño como dientes o individuos fetales han sido documentados.

Los animales de La Cruceta fueron claramente procesados para el consumo. Encontramos marcas de carnicería en los elementos de la mayoría de las especies (Tabla IV), incluso en los restos de équido. También son numerosas las marcas de fracturación, de origen antrópico y realizadas con herramientas contundentes. La mayoría de las marcas se encuentran sobre ovejas y cabras debido a su dominio en el depósito, pero otras especies con menor representación también tienen trazas. Solo se ha realizado un estudio más exhaustivo en ovejas y cabras (Fig. 9). Las trazas de carnice- ría y los patrones de fracturación han sido analizados conjuntamente ya que siguen una misma estrategia de explotación. Se localizan tareas de descuartizado en el cráneo, la escápula, la pelvis, el radio y la ulna, la tibia y las vértebras, que aparecen fracturadas de forma longitudinal por un instrumento contundente. Por otro lado, se localizan tareas de evisceración en la mandíbula y las costillas, y de desmembrado en la pelvis, el astrágalo y el metacarpo. También se detectan marcas de descarnado en los extremos proximales de radio-ulna y tibia. Por último, resulta llamativa la fracturación de la clavija ósea en su base con el objetivo de separarla del cráneo y utilizar el estuche córneo (Fig. 10). El procesado carnicero muestra todos los procesos salvo el

\begin{tabular}{|l|c|c|c|c|c|}
\cline { 2 - 6 } \multicolumn{1}{c|}{} & Total & Fracturas & \%Fracturas & Trazas & $\%$ Trazas \\
\hline Ovis/Capra & 211 & 70 & $33,20 \%$ & 23 & $10,90 \%$ \\
\hline Sus domesticus & 12 & 4 & $33,30 \%$ & & \\
\hline Bos taurus & 4 & 3 & $75,00 \%$ & 1 & $25,00 \%$ \\
\hline Equus sp & 2 & 0 & $0,00 \%$ & 1 & $50,00 \%$ \\
\hline Cervus elaphus & 6 & 2 & $33,30 \%$ & 1 & $16,70 \%$ \\
\hline Oryctolagus cuniculus & 8 & 3 & $37,5 \%$ & & \\
\hline Galliforme & 4 & & & 1 & $25,00 \%$ \\
\hline M. grande & 12 & 6 & $50,00 \%$ & & \\
\hline M. medio-grande & 2 & 1 & $50,00 \%$ & & \\
\hline M. medio & 204 & 72 & $35,30 \%$ & 6 & $2,90 \%$ \\
\hline M. medio-pequeño & 1 & 1 & $100,00 \%$ & 1 & $100,00 \%$ \\
\hline M. pequeño & 1 & 1 & $100,00 \%$ & & \\
\hline
\end{tabular}

Tabla 4: Distribución de las fracturas y trazas antrópicas en las distintas especies del sitio. / Distribution of butchering marks in the different species of the site.

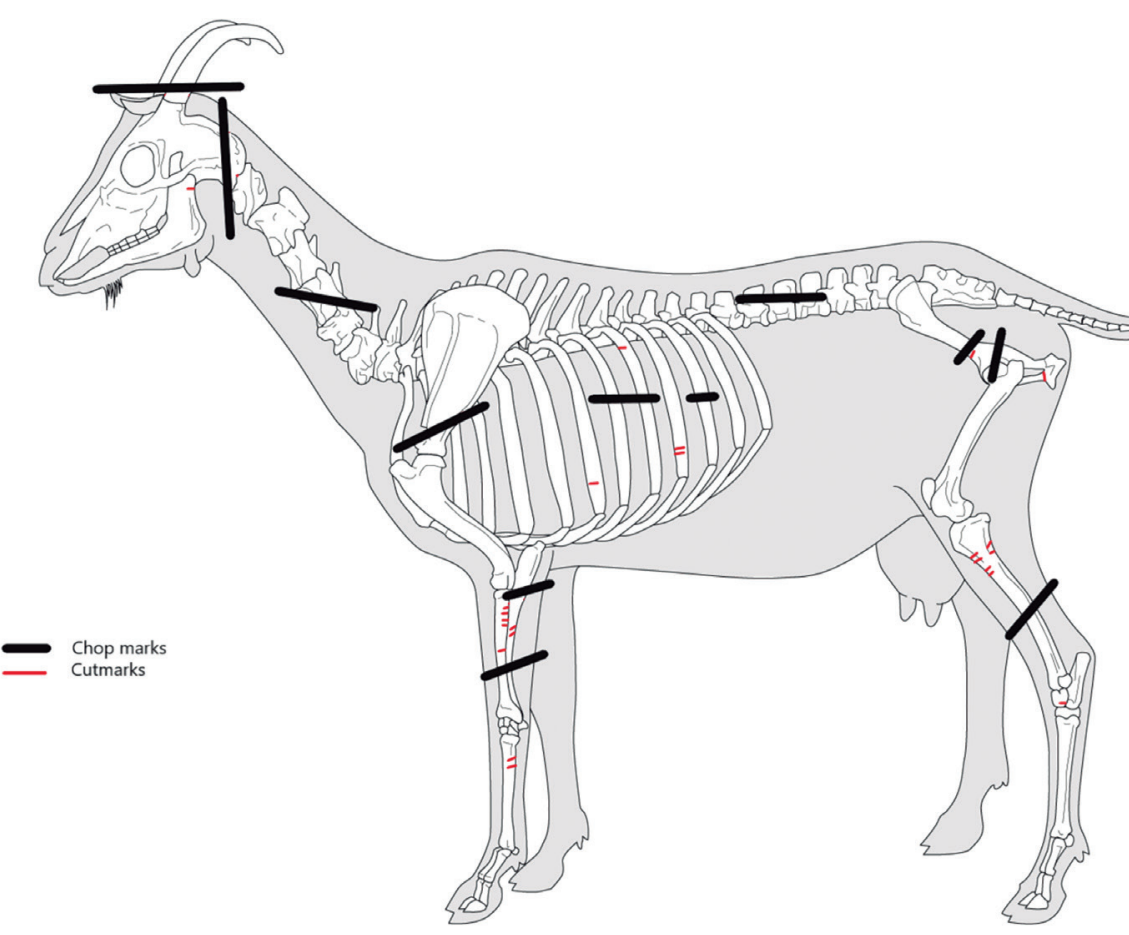

Fig.9. Trazas de procesado (rojo) y de fractura (negro) de ovejas y cabras. / Cutmarks (red) and fracture (black) of sheep and goats. 


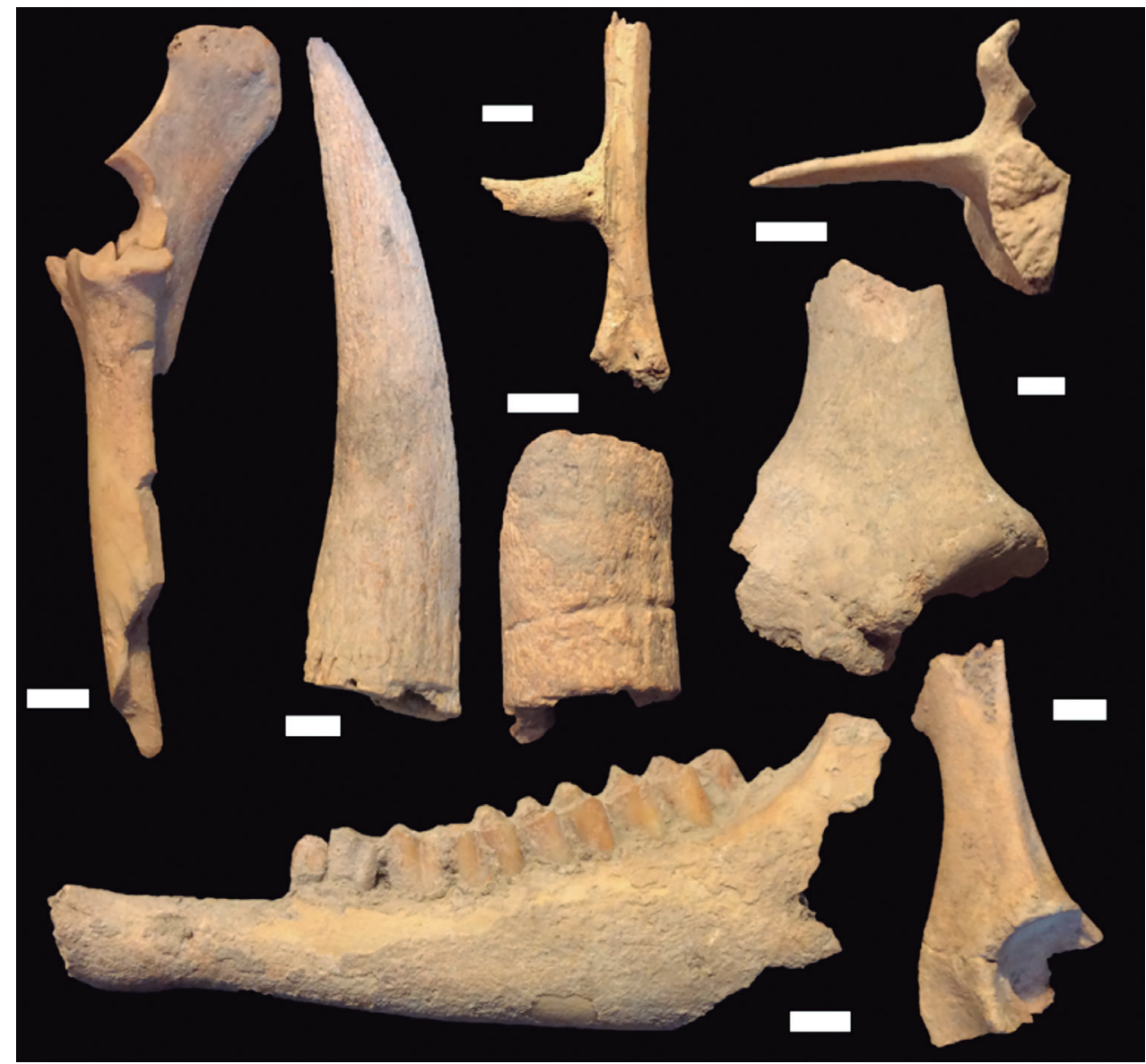

Fig.10. Algunos restos faunísticos de La Cruceta con trazas de corte y marcas de fractura. Línea blanca equivale a $1 \mathrm{~cm}$. / Some faunal remains from La Cruceta with butchering marks. White line corresponds to $1 \mathrm{~cm}$. despellejado. Parece que los individuos se evisceraban y descuartizaban dividiendo en dos mitades la carcasa, como indican las fracturas de las vértebras, y eliminando las partes inferiores. En ninguno de los restos se documentan termoalteraciones.

\section{DISCUSIÓN Y CONCLUSIONES}

El estudio de los restos faunísticos en yacimientos arqueológicos pertenecientes a sociedades complejas ayuda a responder importantes cuestiones sobre estas sociedades, desde las prácticas ganaderas de estos grupos, su alimentación e incluso su estatus socioeconómico y su identidad religiosa (Crabtree, 1990; DeFrance, 2009; Twiss, 2012). En concreto, los habitantes de La Cruceta, aproximadamente desde comienzos del siglo XIII hasta la mitad del XIV, tenían una ganadería centrada en la explotación del ganado ovino y caprino, sobre todo en este último. El dominio de estas dos especies se observa durante la mayor parte de la Edad Media en la Península Ibérica, pero sobre todo a partir del siglo XIII, cuando forma el 50\% de la cabaña doméstica (Grau, 2017). Además del ganado ovino y caprino, el ganado porcino, el vacuno, el equino y el avícola también conformaban la cabaña ganadera de La Cruceta, aunque con mucha menor importancia cuantitativa. Por último, la caza de especies como el ciervo o el conejo también formaba parte de las actividades económicas del sitio. Comparando con otros yacimientos medievales de la mitad norte de la Península Ibérica, las ovejas y las cabras son tan abundantes como en las fases islámicas del castillo de Albarracín (2004) o la judería de Tarrega (Valenzuela-Lamas et al. 2014). En general, el ganado ovino y caprino es más abundante en los castillos que en contextos urbanos. La cabaña ganadera de La Cruceta es similar respecto a otros sitios de funcionalidad militar del noreste peninsular, como el Desolado de Rada (Castaños y Castaños 2003) y el Castell del Mur (s. XV; Valenzuela y Colominas 2009), pero es menor al yacimiento islámico de Zafranales (1988). La abundancia de la oveja y la cabra en la Baja Edad Media se ha relacionado con la producción de lana (Grau 2017), aunque este producto no era especialmente explotado en La Cruceta.

Aunque centrada en el ganado ovino y caprino, la comunidad que ocupaba La Cruceta explotaba una gran diversidad de productos animales. El análisis de las pautas de mortalidad del ganado ovino indica que esta especie fue sacrificada entre los 6 y 24 meses, posiblemente con el objetivo de explotar la carne en el momento del óptimo cárnico (Payne, 1973; Vigne y Helmer, 2007; Blaise, 2009). En contraste, siguiendo 


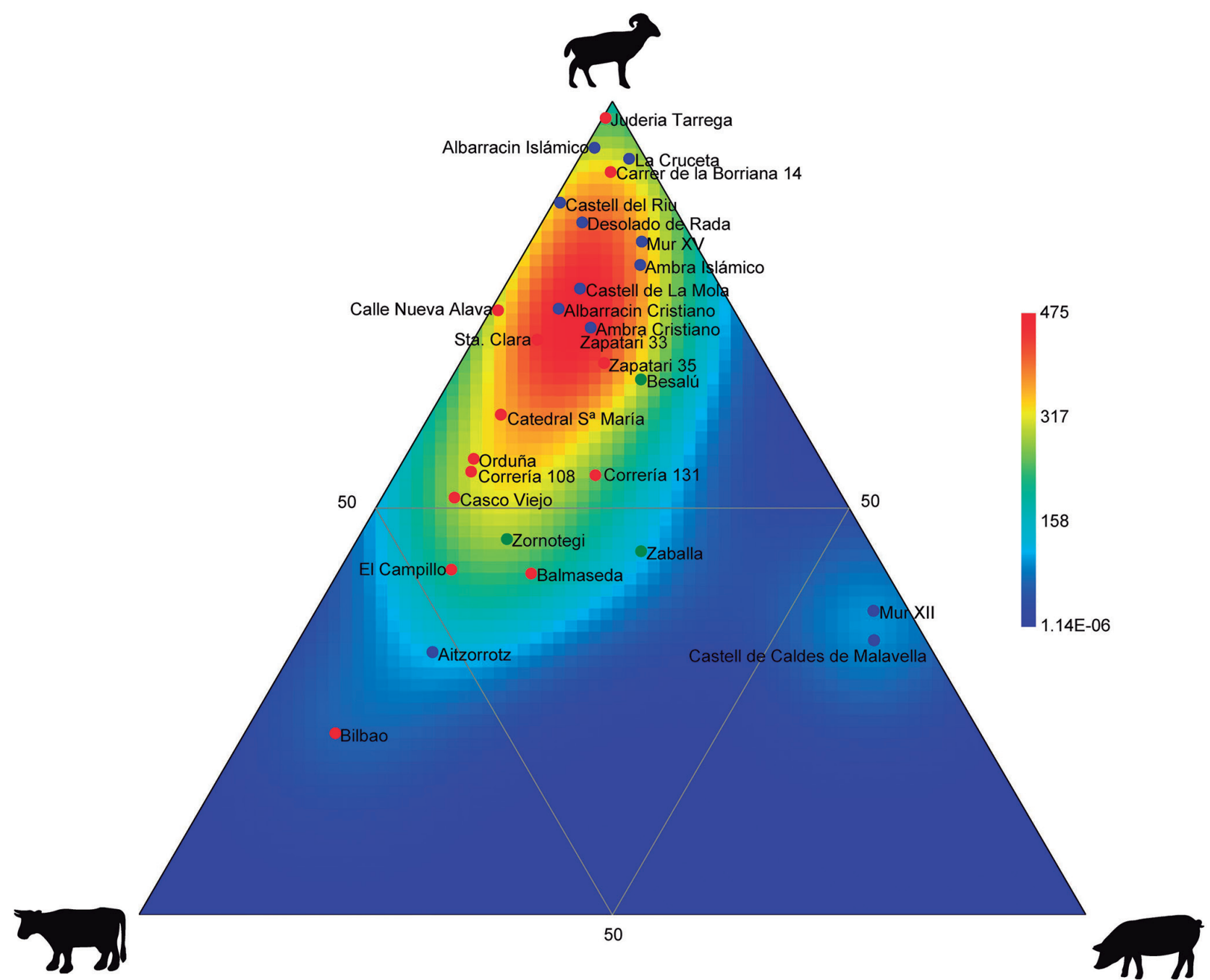

Fig.11. Ternary plot de distintos yacimientos medievales del norte de la Península Ibérica. / Ternary plot from different medieval sites in the north of the Iberian Peninsula.

los mismos modelos, el ganado caprino sería explotado con el objetivo de obtener carne y productos lácteos, debido al sacrificio de los individuos entre los 2 y los 6 meses, justo después del destete, y entre los 24 y 48 meses, por el sacrificio de las hembras que han disminuido su producción láctea (Helmer y Vigne, 2004; Vigne y Helmer, 2007; Blaise, 2009). Además de la carne y la leche, el ganado ovino y caprino es explotado para la obtención de otros productos como materias primas óseas, a través de la extracción del estuche córneo para la realización de diferentes herramientas, y posiblemente las pieles. Por último, no se documenta la un patrón de aprovechamiento especializado de la lana, ya que están ausentes los individuos de edad avanzada en el perfil de mortalidad. La lana era un producto muy apreciado en el reino de Aragón durante Edad Media (Ledesma, 1989; Sarasa, 1989), por lo que la ausencia de documentación de su explotación especializada podría indicar la utilización de este asentamiento para otras actividades económicas. La ausencia de algunos elementos óseos, a pesar de que tienen una alta den- sidad ósea, permite plantear un tratamiento distinto de los mismos. Destaca la ausencia de elementos como húmeros y fémures, con un alto contenido cárnico. Este hecho lleva a plantear la hipótesis una gestión distinta de estos elementos con objetivos de explotación de la carne, bien sea dentro del propio yacimiento o quizá a otro asentamiento (Crabtree, 1990).

Además de los procedentes del ganado ovino y caprino, en La Cruceta se explotaron productos de otras especies. A pesar de la escasez, podemos suponer que se explotaron productos procedentes del ganado vacuno y porcino, especies que serían criadas en el propio sitio (seguramente en el entorno de alguna de las estancias en que han sido halladas), como indica la presencia de animales perinatales, que corresponderían a abortos o animales muertos pocos días después de haber nacido. Los équidos, además de para el transporte, también fueron procesados, por las marcas de corte localizadas. Los productos cinegéticos también eran aprovechados, como es el caso del ciervo. Por último, los productos avícolas son también explo- 
tados, al menos los galliformes, donde se encuentran marcas de corte. Posiblemente, los huevos serían otro producto explotado de estos animales.

En conclusión, nuestra hipótesis es, que además de ser un asentamiento estratégico para el control del área (Gutiérrez González et al., 2017 y 2019), La Cruceta fue también un asentamiento con producción ganadera para el autoconsumo de la comunidad que habitaba el sitio y quizá para el abastecimiento (fundamentalmente de carne) de algún asentamiento cercano al sitio. Esta hipótesis se apoya en la centralización de la producción en el ganado ovino y caprino, con el $80 \%$ de la representatividad, frente a la tendencia peninsular para el mismo periodo (50-60\%) (Grau, 2017). Esto nos lleva a pensar en la existencia de una ganadería orientada a la cría de estas dos especies (Crabtree 1990; García-García, 2017). El perfil de mortalidad de ambas especies, que se corresponde con la explotación de la carne del ganado ovino, con ausencia de explotación especializada de la lana, y de la leche del ganado caprino. Por último, la representación de las regiones anatómicas, que muestra una ausencia destacable de partes como los húmeros y los fémures, elementos con mayor contenido cárnico de estas especies.

La cercanía de La Cruceta con asentamientos como Castejón de Valdejasa o el Castillo de Sora, y su posible dependencia de una institución (Hospital de Santa Cristina, Ios Luna o Tauste) (Gutiérrez González et al., 2017, 2019), refuerzan la idea de que, además de un asentamiento estratégico, se tratara de un asentamiento subsidiario que abastecería, quizá con sus excedentes, a cualquiera de estos sitios, o incluso al municipio de Tauste.

\section{AGRADECIMIENTOS}

La excavación de La Cruceta (Tauste, Zaragoza) es, ante todo, el resultado del interés y el esfuerzo de la Asociación Cultural "El Patiaz" de Tauste, que no ha cejado en su empeño de promover y financiar las variadas intervenciones arqueológicas realizadas. Por ello, no podemos más que agradecer su soporte. También nos gustaría agradecer a los dos revisores por sus oportunas sugerencias que han ayudado a mejorar este texto.

\section{BIBLIOGRAFÍA}

Azorit, C., Analla, M., Carrasco, R., Calvo, J.A., Muñoz-Cobo, J., 2002. Teeth eruption pattern in red deer (Cervus elaphus hispanicus) in southern Spain. Anales de biología 24, 107-114.

Balasse, M., Ambrose, S.H., 2005. Distinguishing sheep and goats using dental morphology and stable carbon isotopes in C4 grassland environments. Journal of Archaeological Science 32(5), 691-702. https://doi.org/10.1016/J.JAS.2004.11.013

Barone, R., 1976. Anatomie comparée des mammifères domestiques. Vigot, Paris.

Binford, L.R., 2014. Bones: ancient men and modern myths. Academic Press, New York.
Blaise, E., 2009. Economie animale et gestion des troupeaux au Néolithique final en Provence: approche archéozoologique et contribution des analyses isotopiques de l'émail dentaire Tesis doctoral inédita. Université de Provence-Aix-Marseille I, Marsella.

Boessneck, J., 1969. Osteological differences between sheep (Ovis aries Linné) and goat (Capra hircus Linné). In: BrothweII, D., Higgs, E., (Eds.), Science in archaeology. A survey in progress and research, 331-358. Thames \& Hudson, London.

Boessneck, J., Müller, H.-H., Teichert, M., 1964. Osteologische Untercheidungsmerkmale zeischen Schaf (Ovis aries Lineé) und Ziege (Capra hircus Lineé). Kühn Archive 78, 1-119.

Brown, W.A.B., Chapman, N.G., 1991. Age assessment of red deer (Cervus elaphus): from a scoring scheme based on radiographs of developing permanent molariform teeth. Journal of Zoology 225(1), 85-97.

Castaños, P., Castaños, J., 2003. Estudio de la fauna del yacimiento medieval de Desolado de Rada (Navarra). Kobie Paleoantropología 27, 205-230.

Castaños, P., 1988. Estudio de los restos faunísticos del yacimiento de Zafranales. Annales: Anuario del Centro de la Universidad Nacional de Educación a Distancia de Barbastro 5, 147-162.

Cohen, A., Serjeantson, D., 1996. A manual for the identification of bird bones from archaeological sites. Archetype Publications, London.

Crabtree, P.J., 1990. Zooarchaeology and complex societies: some uses of faunal analysis for the study of trade, social status, and ethnicity. Archaeological method and theory 2, 155-205.

DeFrance, S.D., 2009. Zooarchaeology in complex societies: political economy, status, and ideology. Journal of archaeological research 17, 105-168.

Díez, J.C., 1992. Zooarqueología de Atapuerca (Burgos) e implicaciones paleoeconómicas del estudio tafonómico de yacimientos del Pleistoceno Medio. Unpublished PhD thesis. Universidad Complutense de Madrid, Madrid.

Falcón, I., 1998. Aprovisionamiento y sanidad en Zaragoza en el siglo XV. Acta historica et archaeologica mediaevalia 19, 127-144.

Fernández Otal, J.A., 1996. La Casa de Ganaderos de Zaragoza en la Edad Media:(siglos XIII-XV): aportación a la historia pecuaria del Aragón medieval. Prensas Universitarias de Zaragoza.

Fernandez, H., 2001. Ostéologie comparée des petits ruminants eurasiatiques sauvages et domestiques (genres Rupicapra, Ovis, Capra et Capreolus): diagnose différentielle du squelette appendiculaire. Tesis doctoral. Universidad de Ginebra, Ginebra.

García-García, M., 2017. Some remarks on the provision of animal products to urban centres in medieval Islamic Iberia: The cases of Madinat Ilbirah (Granada) and Cercadilla (Cordova). Quaternary International 460, 86-96.

García-García, M., 2017. El abastecimiento urbano de productos animales en al-Andalus: reflexiones en torno a su estudio arqueológico. In: Villar Mañas, S., García-García, M. (Eds.), Ganadería y arqueología medieval, 73-98. Alhulia, Granada.

Gerbault, P., Gillis, R., Vigne, J.D., Tresset, A., Bréhard, S., Thomas, M.G., 2016. Statistically robust representation and comparison of mortality profiles in archaeozoology. Journal of Archaeological Science 71, 24-32. https://doi.org/10.1016/j. jas.2016.05.001 
Gillis, R., Chaix, L., Vigne, J.D., 2011. An assessment of morphological criteria for discriminating sheep and goat mandibles on a large prehistoric archaeological assemblage (Kerma, Sudan). Journal of Archaeological Science 38(9), 2324-2339. https://doi.org/10.1016/j.jas.2011.04.012

Grant, A., 1982. The use of tooth wear as a guide to the age of domestic animals. In: Wilson, B., Grigson, C., Payne, S. (Eds.), Ageing and Sexing Animal Bones from Archaeological Sites, 91-108. B.A.R., Oxford.

Grau, I., García-Garcia, M., 2018. Zooarqueología y Edad Media en la Península Ibérica. In: Quirós Castillo, J. A., Treinta años de arqueología medieval en España, 341-364. Archaeopress.

Grau-Sologestoa, I., 2017. Socio-economic status and religious identity in medieval Iberia: The zooarchaeological evidence. Environmental Archaeology 22(2), 189-199.

Grau-Sologestoa, I., Albarella, U., Castillo, J.A.Q., 2016. Urban medieval and post-medieval zooarchaeology in the Basque Country: Meat supply and consumption. Quaternary International 399, 1-12.

Gutiérrez González, F.J., Lafuente Gómez, M. Lanzas Orensanz, O., Gil de Muro Eguizábal, V., Valladares Lafuente, C., 2018. La Cruceta de Tauste. Primeras investigaciones de un puesto de vigilancia bajomedieval. In: VVAA, II Congreso Aragonés de Arqueología y Patrimonio, noviembre 2017, 377-387. Zaragoza.

Gutiérrez González, F.J., Lafuente Gómez, M., Lanzas Orensanz, O., Gil de Muro Eguizábal, V., Valladares Lafuente, C., Sierra Sainz-Aja, A., 2019. La Cruceta de Tauste. Arqueología e Historia de un asentamiento bajomedieval. In: VVAA, Tauste en su historia. Actas de las XIX Jornadas sobre Historia de Tauste, febrero-marzo de 2018, 5-62. Asociación Cultural el Patiaz, Tauste.

Halstead, P., Collins, P., Isaakidou, V., 2002. Sorting the sheep from the goats: Morphological distinctions between the mandibles and mandibular teeth of adult Ovis and Capra. Journal of Archaeological Science 29(5), 545-553. https://doi. org/10.1006/jasc.2001.0777

Helmer, D., 1995. Biometria i arqueozoologia a partir d'alguns exemples del Proxim Orient. Cota zero: Revista d'arqueologia i ciència 11, 51-60.

Helmer, D., 2000. Discrimination des genres Ovis et Capra à l'aide des prémolaires inférieures 3 et 4 et interprétation des âges d'abattage: l'exemple de Dikili Tash (Grèce). Anthropozoologica 31, 29-38.

Helmer, D., Vigne, J.D., 2004. La gestion des cheptels de caprinés au Néolithique dans le midi de la France. In: Bodu, P., Constantin, C. (Dir.), Approches fonctionnelles en Préhistoire. Actes XXVe Congres Préhistorique de France Nanterre, 24-26 novembre 2000, 397-407. Société Préhistorique Française Édition, Paris.

Ioannidou, E., 2003. Taphonomy of animal bones: species, sex, age and breed variability of sheep, cattle and pig bone density. Journal of Archaeological Science 30(3), 355-365.

Lavocat, R., 1966. Atlas de prehistoire, volume 3: Faunes et flores préhistoriques de l'Europe occidentale. N. Boubée, Paris.

Ledesma, M.L., 1989. La ocupación del espacio y las transformaciones económicas en Aragón en los siglos XII y XIII. In: VVAA, Historia de Aragón, 75-86. Institución Fernando el Católico.

Legge, A.J., 1992. Excavations at Grimes Graves Norfolk 1972-1976 Fascicule 4: Animals, Environment and the Bronze Age Economy. British Museum Press, London.
Lemoine, X., Zeder, M.A., Bishop, K.J., Rufolo, S.J., 2014. A new system for computing dentition-based age profiles in Sus scrofa. Journal of Archaeological Science 47, 179-193. https:// doi.org/10.1016/J.JAS.2014.04.002

Lyman, R.L., Lyman, C., 1994. Vertebrate taphonomy. Cambridge University Press.

Mariezkurrena, K., Altuna, J., 1983. Contribución al conocimiento del desarrollo de la dentición y el esqueleto poscraneal de Cervus elaphus. Munibe Antropologia-Arkeologia 35(3-4), 149-202.

Moreno-García, M., 2004. Musulmanes y cristianos en la Sierra de Albarracín (Teruel): una contribución desde la Arqueozoología para la historia de la trashumancia. In: La trashumancia en la España mediterránea: historia, antropología, medio natural, desarrollo rural, 233-262. Rolde de estudios aragoneses.

Moreno-García, M., 1999, The zooarchaeology of transhumance in medieval Spain. Tesis de doctorado inédita, University of Cambridge.

Navarro, G., 2003. La industria textil en los reinos de Aragón y Valencia en la Edad Media. In: Claramunt Rodríguez, S. (Coord.), Actas del XVII Congreso de Historia de la Corona de Aragón Vol. 1, 475-491. Universidad de Barcelona.

Pales, L., Lambert, C., Garcia, M.-A., 1971. Atlas ostéologique pour servir à l'identification des mammifères du quaternaire. Editions du Centre national de la recherche scientifique, Paris.

Payne, S., 1973. Kill-off Patterns in Sheep and Goats: the Mandibles from Aşvan Kale. Anatolian Studies 23, 281-303. https:// doi.org/10.2307/3642547

Payne, S., 1985. Morphological distinctions between the mandibular teeth of young sheep, Ovis, and goats, Capra. Journal of Archaeological Science 12(2), 139-147. https://doi. org/10.1016/0305-4403(85)90058-5

Payne, S., 1987. Reference codes for wear states in the mandibular cheek teeth of sheep and goats. Journal of Archaeological Science 14(6), 609-614. https://doi.org/10.1016/03054403(87)90079-3

Pérez-Ripoll, M., 1992. Marcas de carnicería, fracturas intencionadas y mordeduras de carnívoros: en huesos prehistóricos del mediterráneo español (Vol. 15). Instituto de Cultura "Juan Gil-Albert".

Potts, R., Shipman, P., 1981. Cutmarks made by stone tools on bones from Olduvai Gorge, Tanzania. Nature 291(5816), 577-580.

Prummel, W., Frisch, H.J., 1986. A guide for the distinction of species, sex and body side in bones of sheep and goat. Journal of Archaeological Science 13(6), 567-577. https://doi. org/10.1016/0305-4403(86)90041-5

Saña, M., 1999. Arqueología de la domesticación animal: la gestión de los recursos animales en Tell Halula (Valle del Éufrates, Siria): del 8.800 al 7.000 BP. Treballs d'Arqueologia del Pròxim Orient. Universitat Autònoma de Barcelona, Barcelona.

Sarasa, E., 1989. Las actividades económicas en una época de crisis (siglos XIV-XV). In: VVAA, Historia de Aragón, 87-100. Institución Fernando el Católico.

Sarasa, E., 1995. Los sistemas alimentarios en el reino de Aragón (siglos XII-XV). In: VVAA (Eds.), Actes - I Col.loqui d'Història de l'Alimentació a la Corona d'Aragó: Edat Mitjana, 185204. Institut d'Estudis llerdencs.

Schmid, E., 1972. Atlas of animal bones for prehistorians, archaeologists, and Quaternary geologists. Elsevier Science Publishers, Amsterdam. 
Sénac, P., 2009. Un "village" d'Al-Andalus aux alentours de l'an mil: Las Sillas (Marcén, province de Huesca). Université de Tolouse-Le Mirail.

Sesma, J.Á., 1977. El comercio de exportación de trigo, aceite y lana desde Zaragoza a mediados del siglo XV. Aragón en la Edad Media 1, 201-237.

Sesma, J.Á., 1995. Producción para el mercado, comercio y desarrollo mercantil en espacios interiores (1250-1350): el modelo del sur de Aragón. In: Europa en los umbrales de la crisis, 1250-1350: [Actas de la] XXI Semana de Estudios Medievales, Estella, 18 a 22 de julio de 1994, 205-246. Departamento de Educación y Cultura.

Sesma, J.Á., 2010. El comercio exterior de la corona por vías terrestres. Un comercio intenso y fragmentado. In: Sesma, J.Á. (coord), La Corona de Aragón en el centro de su historia, 1208-1458: aspectos económicos y sociales:[congreso] Zaragoza y Calatayud, 24 al 26 de noviembre de 2009, 345-362. Universidad de Zaragoza.

Silver, I.A., 1969. The ageing of domestic animals. In: BrothweII, D., Higgs, E., (Eds.), Science in archaeology. A survey in progress and research, 283-302. Thames \& Hudson, London.

Valenzuela, S., Colominas, L., 2009. Analisi de la gestio dels recursos animals al castell de Mur. In: Sancho, M. (Ed.), Mur, la Historia d'un Castell Feudal a la llum de la recerca histórica-arqueológica, Garsineu Edicions.

Valenzuela-Lamas, S., Valenzuela-Suau, L., Saula, O., Colet, A., Mercadal, O., Subiranas, C., Nadal, J., 2014. Shechita and Kashrut: identifying Jewish populations through zooarchaeo-

\section{ANEXO: Medidas}

\begin{tabular}{|l|c|c|c|c|l|l|}
\hline & Clavija & A & L & & & \\
\hline CAHI & & 27.6 & 18.5 & & & \\
\hline CAHI & & 26.6 & 18.5 & & & \\
\hline & & & & & & \\
\hline OC & Escápula & GLP & BG & LG & & \\
\hline OC & & 23.6 & 17.2 & & & \\
\hline & & & 21.1 & 25.4 & & \\
\hline OVAR & & 30.6 & 27.9 & & & \\
\hline CAHI & & 25.2 & 24.4 & & & \\
\hline OVAR & & 31.2 & 28.3 & & & \\
\hline & & & & & & \\
\hline & Ulna & BPC & LO & DPA & SDO & \\
\hline OC & & 18.9 & 36.3 & 24.7 & 20.8 & \\
\hline OC & & 18.2 & & & & \\
\hline & & & & & & \\
\hline & Tibia & BD & DD & & & \\
\hline CAHI & & 27.1 & 20.6 & & & \\
\hline CAHI & & 23.9 & 17.9 & & & \\
\hline OVAR & & 25.3 & 19.7 & & & \\
\hline OVAR & & 25 & 17.3 & & & \\
\hline CAHI & & 24.9 & 19 & & & \\
\hline & & & & & & \\
\hline
\end{tabular}

logy and taphonomy. Two examples from Medieval Catalonia (North-Eastern Spain). Quaternary International 330, 109-117.

Varela, S., Rodríguez, J., 2004. Atlas osteológico, carnívoros ibéricos. MNCN, Madrid.

Vigil-Escalera Guirado, A., Moreno-García, M., Peña-Chocarro, L., Morales Muñiz, A., Llorente Rodríguez, L., Sabato, D., Ucchesu, M., 2014. Productive strategies and consumption patterns in the Early Medieval village of Gózquez (Madrid, Spain). Quaternary International 346, 7-19.

Vigne, J.D., Helmer, D., 2007. Was milk a "secondary product" in the Old World Neolithisation process? Its role in the domestication of cattle, sheep and goats. Anthropozoologica 42(2), 9-40.

Villa, P., Mahieu, E., 1991. Breakage patterns of human long bones. Journal of human evolution 21(1), 27-48.

Zeder, M.A., Lapham, H.A., 2010. Assessing the reliability of criteria used to identify postcranial bones in sheep, Ovis, and goats, Capra. Journal of Archaeological Science 37(11), 28872905. https://doi.org/10.1016/j.jas.2010.06.032

Zeder, M.A., Lemoine, X., Payne, S., 2015. A new system for computing long-bone fusion age profiles in Sus scrofa. Journal of Archaeological Science 55, 135-150. https://doi.org/10.1016/J.JAS.2014.12.017

Zeder, M.A., Pilaar, S.E., 2010. Assessing the reliability of criteria used to identify mandibles and mandibular teeth in sheep, Ovis, and goats, Capra. Journal of Archaeological Science 37(2), 225-242. https://doi.org/10.1016/j.jas.2009.10.002.

\begin{tabular}{|c|c|c|c|c|c|c|}
\hline & Astrágalo & GLL & GLM & $\mathrm{BD}$ & $\mathrm{DL}$ & DM \\
\hline OVAR & & 27.4 & 26.5 & 18.4 & 15 & 15.4 \\
\hline CAHI & & 27.3 & 25.9 & 17.3 & 14.8 & 15.4 \\
\hline \multirow[t]{2}{*}{ CAHI } & & 25.5 & 29.8 & 16.9 & 13.2 & 15.3 \\
\hline & Calcaneo & $\mathrm{GH}$ & GB & BFD & LMT & \\
\hline \multirow[t]{2}{*}{ EQCA } & & 56.1 & 61 & 48.8 & 57.6 & \\
\hline & Metatarso & $\mathrm{BP}$ & SD & & & \\
\hline \multirow[t]{2}{*}{ oc } & & 17.3 & 8.8 & & & \\
\hline & Metacarpo & $\mathrm{BP}$ & & & & \\
\hline \multirow[t]{2}{*}{ OC } & & 22.3 & & & & \\
\hline & Falange 1 & $\mathrm{GL}$ & GLPE & $\mathrm{BP}$ & $\mathrm{BD}$ & \\
\hline CEEL & & 46.3 & 47.3 & 16.3 & 15.5 & \\
\hline OVAR & & 34.5 & 34.9 & 12.1 & 11.1 & \\
\hline SUDO & & 35.1 & 34.9 & 15.1 & 15.4 & \\
\hline
\end{tabular}


